\title{
NULIITY DISTRIBUTION ON THE TANGENT BUNDLE OF A RIEMANNIAN MANIFOLD
}

\author{
WEE-KEONG LIM
}

\begin{abstract}
The nullity distribution on the tangent bundle of a Riemannian manifold is studied. The vetical and complete lifts of a vector fileld belonging to the nullity distribution on the base manifold are show to be in the nullity distribution on the tangent bundle. Under certain regularity assumption, the dimension of the nullity distribution on the tangent bundle of a Riemannian manifold is found to be twice that of the nullity distribution on the base manifold.
\end{abstract}

\section{Introduction}

Let $(M, g)$ be a $C^{\infty}$ Riemannian $n$-manifold and $T_{p} M$ be its tangent space at the point $p \in M$. The tangent bundle $T M$ of $M$ is defined to be the union of all tangent spaces over $M$,

$$
T M=\bigcup_{p \in M} T_{p} M
$$

The projection map $\pi: T M \rightarrow M$ is defined by $\pi(X)=p$ for all $X \in T_{p} M$. We will write $(p, X)$ for $X \in T_{p} M$.

Let $(\phi, U)$ be a coordinate chart on $M$ and $\left(x^{1}, x^{2}, \ldots, x^{n}\right)$ be the coordinate system on $U$. Define $\bar{x}^{i}=x^{i} \circ \pi, \bar{y}^{i}(p, X)=X^{i}$ where $X=\sum_{k=1}^{n} X^{i}\left(\frac{\partial}{\partial x^{i}}\right)_{p},(p, X) \in T_{p} M$. Then $\bar{\phi}=\left(\bar{x}^{1}, \bar{x}^{2}, \ldots, \bar{x}^{n}, \bar{y}^{1}, \bar{y}^{2}, \ldots, \bar{y}^{n}\right)$ defines a coordinate system on $\pi^{-1}(U)$. We will call this the induced coordinate system. We will sometimes write $\left(\bar{x}^{1}, \bar{x}^{2}, \ldots, \bar{x}^{n}, \bar{y}^{1}, \bar{y}^{2}, \ldots, \bar{y}^{n}\right)$ as $\left(\bar{x}^{1}, \bar{x}^{2}, \ldots, \bar{x}^{n}, \bar{x}^{n+1}, \bar{x}^{n+2}, \ldots, \bar{x}^{2 n}\right)$ or $\left(\bar{x}^{1}, \bar{x}^{2}, \ldots, \bar{x}^{n}, \bar{x}^{\overline{1}}, \bar{x}^{2}, \ldots, \bar{x}^{\bar{n}}\right)$.

A 1 -form $\omega$ in $M$ can be viewed as a function in TM, which will be denoted by $\imath \omega$. If $\omega=\sum_{i=1}^{n} \omega_{i} d x^{i}$ then $\imath \omega=\sum_{i=1}^{n} \omega_{i} \bar{y}^{i}$.

Let $\Im_{s}^{r}(M)$ denotes the set of all tensor fields of type $(r, s)$ on $M$ and $\Im(M)=$ $\sum_{r, s=0}^{\infty} \Im_{s}^{r}(M)$. Note that $\Im_{0}^{0}(M)=C^{\infty}(M)$ and $\Im_{0}^{1}(M)$ is the set of all $C^{\infty}$ vector fields on $M$.

Unless it is specifically mentioned, the summation range for $h, i, j, k, l$ are from $1,2, \ldots, n$, and for $A, B$ are from $1,2, \ldots, n, \overline{1}, \overline{2}, \ldots, \bar{n}$.

The nullity distribution was first defined by Chern and Kuiper in their paper [1] on a Rimannian manifold $(M, g)$. The distribution assigns to each point $m \in M$, the subspace

$$
N(m)=N_{m}=\left\{X \in T_{m} M \mid R(X, Y)=0, \quad \text { for all } Y \in T_{m} M\right\}
$$

Received October 2, 1997.

1991 Mathematics Subject Classification. 53xx.

Key words and phrases. Nullity distribution, tangent bundles. 
where $\nabla$ is the Riemannian connection with respect to $g$ in $M$ and $R$ is the curvature tensor. We denote the nullity distribution by $N$. If $U$ is an open set in $M$, then $\Gamma(U, N)$ denotes the set of all vector fields $X$ such that $X(m) \in N_{m}$ for any $m \in U$. When $U=M$, we write $\Gamma(N)$ for $\Gamma(M, N)$. [3].

In the same way, the nullity distribution on an affine manifold can also be cansidered

We have the following simple characterization of a nullity distribution which we will need in the sections to follow.

Proposition 1. A vector field $X=\sum_{k=1}^{n} X^{k} \frac{\partial}{\partial x^{k}}$ on $M$ belongs to $\Gamma(N)$ if and only if $\sum_{k}^{n}{ }_{k=1} X^{k} R_{k j i}{ }^{h}=0$ for all $h, i, j=1,2, \ldots, n$ where $R_{k j i}{ }^{h}$ are the local components of we have

Proof. Since $X \in \Gamma(N)$, then for all $Y=\sum_{i=1}^{n} Y^{i} \frac{\partial}{\partial x^{i}}, Z=\sum_{i=1}^{n} Z^{i} \frac{\partial}{\partial x^{i}} \in \Im_{0}^{1}(M)$,

$$
R(X, Y) Z=\sum_{h, i, j, k=1}^{n} X^{k} Y^{j} Z^{i} R_{k j i}{ }^{h} \frac{\partial}{\partial x^{h}}=0 .
$$

Thus $\sum_{h, k=1}^{n} X^{k} R_{k j i}{ }^{h} \frac{\partial}{\partial x^{h}}=0$ which implies that $\sum_{k=1}^{n} X^{k} R_{k j i}{ }^{h}=0$ for all $i, j, h=$
$1,2, \ldots, n$.

\section{Vertical and Complete Lifts}

In the following sections, we will follow closely the definition and terminology in [4].

We define the vertical life of a $C^{\infty}$ function $f$ in $M$ as $f^{V}=f \circ \pi: T M \rightarrow R$, which is also $C^{\infty}$ on $T M$. If there is no confusion, we will write $f^{V}$ as $f$.

The vertical lift of a vector field $X=\sum_{h=1}^{n} X^{h} \frac{\partial}{\partial x^{h}}$ in $M$ is defined to be a vector field in $T M$. written as $X^{V}$, which satisfies $X^{V}(\imath \omega)=(\omega X)^{V}$ for all $\omega \in \Im_{1}^{0}(M)$. Thus $X^{V}=\sum_{h=1}^{n} X^{h} \frac{\partial}{\partial \bar{y}^{h}} \in \Im_{0}^{1}(T M)$.

The complete life of $f \in \Im_{0}^{0}(M)$, written as $f^{C}$, is defined to be $f^{C}=\imath(d f)=$ $\sum_{i=1}^{n} \bar{y}^{i} \frac{\partial f}{\partial \bar{x}^{i}}$. We shall write $\partial f$ for $\sum_{i=1}^{n} \bar{y}^{i} \frac{\partial f}{\partial \bar{x}^{i}} \in \Im_{0}^{0}(T M)$.

The complete life of $X=\sum_{i=1}^{n} X^{i} \frac{\partial}{\partial x^{i}} \in \Im_{0}^{1}(M)$ written as $X^{C}$, is defined by $X^{C} f^{C}=$ $(X f)^{C}$ for any $f \in \Im_{0}^{0}(M)$. Thus $X^{C} \stackrel{\partial x^{i}}{=} \sum_{h=1}^{n}\left(X^{h} \frac{\partial}{\partial \bar{x}^{h}}+\partial X^{h} \frac{\partial}{\partial \bar{y}^{h}}\right) \in \Im_{0}^{1}(T M)$.

The complete lift of a 1-form $\omega=\sum_{i=1}^{n} \omega_{i} d x^{i}$, written as $\omega^{C}$, is defined by $\omega^{C}\left(X^{C}\right)=$ $(\omega X)^{C}$ for any $X \in \Im_{0}^{1}(M)$. Thus $\omega^{C}=\sum_{i=1}^{n}\left(\partial \omega_{i} d \bar{x}^{i}=\omega_{i} d \bar{y}^{i}\right) \in \Im_{1}^{0}(T M)$.

By taking account of

$$
\begin{aligned}
(g f)^{C} & =g^{C} f^{V}+g^{V} f^{C}, \\
(f X)^{C} & =f^{V} X^{C}+f^{C} X^{V}, \\
(f \omega)^{C} & =f^{V} \omega^{C}+f^{C} \omega^{V}
\end{aligned}
$$

where $g, f \in \Im_{0}^{0}(M), X \in \Im_{0}^{1}(M), \omega \in \Im_{1}^{0}(M)$, we extend the definition of complete lift to arbitrary tensor field by imposing the following conditions:

$$
(P \otimes Q)^{C}=P^{C} \otimes Q^{C},
$$




$$
(P+R)^{C}=P^{C}+R^{C}
$$

where $Q$ is any tensor field and $P, R$ are any two tensor fields of the same type.

Now, we look at some special type of tensor fields which will be used later:

(i) A metric in $M, g=\sum_{i, j=1}^{n} g_{j i} d x^{j} \otimes d^{i} \in \Im_{2}^{0}(M)$. Thus

$$
g^{C}=\sum_{i, j=1}^{n}\left(g_{j i} d \bar{x}^{j} \otimes d \bar{y}^{i}+g_{j i} d \bar{y}^{j} \otimes d \bar{x}^{i}+\partial g_{j i} d \bar{x}^{j} \otimes d \bar{x}^{i}\right) .
$$

If $g$ is a Riemannian metric, then $g^{C}$ is a pseudo-Riemannian metric on $T M[4, \mathrm{pg}$ 38].

(ii) $S=\sum_{h, i_{s}, \ldots, i_{1}=1}^{n} S_{i_{s}, i_{s-1}, \ldots, i_{1}}^{h} \frac{\partial}{\partial x^{h}} \otimes d x_{i_{s}} \otimes d x_{i_{s-1}} \otimes \cdots \otimes d x_{i_{1}} \in \Im_{s}^{1}(M)$. Thus

$$
\begin{aligned}
S^{C}= & \sum_{h, i_{s}, i_{s-1}, \ldots, i_{1}}^{n}\left(S_{i_{s}, i_{s-1}, \ldots, i_{1}}^{h} \frac{\partial}{\partial \bar{x}^{h}} \otimes d \bar{x}_{i_{s}} \otimes d \bar{x}_{i_{s-1}} \otimes \cdots \otimes d \bar{x}_{i_{1}}\right. \\
& +\partial S_{i_{s}, i_{s-1}, \ldots, i_{1}}^{h} \frac{\partial}{\partial \bar{y}^{h}} \otimes d \bar{x}_{i_{s}} \otimes d \bar{x}_{i_{s-1}} \otimes \cdots \otimes d \bar{x}_{i_{1}}+\sum_{t=1}^{s} S_{i_{s}, i_{s-1}, \ldots, i_{1}}^{h} \frac{\partial}{\partial \bar{y}^{h}} \\
& \left.\otimes d \bar{x}_{i_{s}} \otimes d \bar{x}_{i_{s-1}} \otimes \cdots \otimes d \bar{x}_{i_{t+1}} \otimes d \bar{y}_{i_{t}} \otimes d \bar{x}_{i_{t-1}} \otimes \cdots \otimes d \bar{x}_{i_{1}}\right) .
\end{aligned}
$$

\section{Nullity Distribution on $\left(T M, g^{C}, \nabla^{C}\right)$}

Let $(M, g, \nabla)$ be a Riemannian manifold. Let $R, T$ be its curvature and torsion tensors with respect to $\nabla$. The tangent bundle $T M$ with metric $g^{C}$, the complete lift of $g$ to $T M$, will form a pseudo-Riemannian manifold. The connection induced by $g^{C}$, will be $\nabla^{C}$ while the curvature and torsion tensor will be $R^{C}$ and $T^{C}$ respectively [4, page 41]. From (1), the components of the curvature tensor $R^{C}$ of $\nabla^{C}, \bar{R}_{A B C}^{D}$, with respect to the induced coordinates in $T M$, where $A, B, C, D=1,2, \ldots, n, \overline{1}, \overline{2}, \ldots, \bar{n}$, can be expressed in term of the components of $R$,

$$
\begin{aligned}
& \bar{R}_{k j i}^{h}=R_{k j i}{ }^{h}, \bar{R}_{k j i}^{\bar{h}}=\partial \bar{R}_{k j \bar{i}}^{h}, \\
& \bar{R}_{k j i}^{\bar{h}}=R_{k j i}{ }^{h}, \bar{R}_{k \bar{j} i}^{\bar{h}}=R_{k j i}{ }^{h}, \bar{R}_{\bar{k} j i}^{\bar{h}}=R_{k j i}{ }^{h},
\end{aligned}
$$

all the others being zero.

Let $\bar{N}$ be the nullity distribtuion in $T M$ with respect to $R^{C}$. If $\bar{X}=\sum_{h=1}^{n}\left(\bar{X}^{h} \frac{\partial}{\partial \bar{x}^{h}}+\right.$ $\left.\bar{X}^{\bar{h}} \frac{\partial}{\partial \bar{x}^{\bar{h}}}\right)$ is in $\Gamma(\bar{N})$, then from Proposition 1,

$$
\sum_{A} \bar{R}_{A B C}^{D} \bar{X}^{A}=0 \quad \text { for any } B, C, D=1,2, \ldots, n, \overline{1}, \overline{2}, \ldots, \bar{n} \text {. }
$$

From (2), the above equations (3) reduce to the following two cases: 
(case 1) $D=h, B=j, C=i$, we have

$$
\sum_{A} \bar{R}_{A j i}^{h} \bar{X}^{A}=\sum_{k=1}^{n} R_{k j i}{ }^{h} \bar{X}^{k}=0 .
$$

(case 2) $D=\bar{h}, B=j, C=i$, we have

$$
\begin{aligned}
0 & =\sum_{A} R_{A j i}^{-{ }^{h}} \bar{X}^{A} \\
& =\sum_{k=1}^{n}\left(\partial R_{k j i}{ }^{h} \bar{X}^{k}+R_{k j i}{ }^{h} \bar{X}^{\bar{k}}\right) \\
& =\sum_{k=1}^{n}\left(\partial R_{k j i}{ }^{h} \bar{X}^{k}+R_{k j i}{ }^{h} \partial \bar{X}^{k}-R_{k j i}{ }^{h} \partial \bar{X}^{k}+R_{k j i}{ }^{h} \bar{X}^{\bar{k}}\right) \\
& =\partial\left(\sum_{k=1}^{n} R_{k j i}{ }^{h} \bar{X}^{k}\right)+\sum_{k=1}^{n} R_{k j i}{ }^{h}\left(\bar{X}^{\bar{k}}-\partial \bar{X}^{k}\right) \\
& =\sum_{k=1}^{n} R_{k j i}{ }^{h}\left(\bar{X}^{\bar{k}}-\partial \bar{X}^{k}\right) .
\end{aligned}
$$

From (4) and (5) above, we have if

Proposition 2. A vector field $\bar{X}=\sum_{h=1}^{n}\left(\bar{X}^{h} \frac{\partial}{\partial \bar{x}^{h}}+\bar{X}^{\bar{h}} \frac{\partial}{\partial \bar{x}^{\bar{h}}}\right)$ is in $\Gamma(\bar{N})$ if and only

$$
\left\{\begin{aligned}
\sum_{k=1}^{n} R_{k j i}{ }^{h} \bar{X}^{k} & =0 \\
\sum_{k=1}^{n} R_{k j i}{ }^{h}\left(\bar{X}^{\bar{k}}-\partial \bar{X}^{k}\right) & =0
\end{aligned}\right\} \quad h, i, j=1,2, \ldots, n .
$$

Proposition 3. For any $X \in \Gamma(N)$, we have $X^{V}, X^{C} \in \Gamma(\bar{N})$.

Proof. Let $X=\sum_{h=1}^{n} X^{h} \frac{\partial}{\partial x^{h}} \in \Gamma(N)$. We have $\sum_{k=1}^{n} R_{k j i}{ }^{h} X^{k}=0$.

For $X^{V}=\sum_{h=1}^{n}\left(\bar{X}^{h} \frac{\partial}{\partial \bar{x}^{h}}+\bar{X}^{\bar{h}} \frac{\partial}{\partial \bar{y}^{h}}\right)=\sum_{h=1}^{n} X^{h} \frac{\partial}{\partial \bar{y}^{h}}$, its components satisfy

$$
\begin{aligned}
\sum_{k=1}^{n} R_{k j i}{ }^{h} \bar{X}^{k} & =0 \quad \text { and } \\
\sum_{k=1}^{n} R_{k j i}{ }^{h}\left(\bar{X}^{\bar{k}}-\partial \bar{X}^{k}\right) & =\sum_{k=1}^{n} R_{k j i}{ }^{h} X^{k} \\
& =0
\end{aligned}
$$

for all $h, i, j=1,2, \ldots, n$. satisfy

For $X^{C}=\sum_{h=1}^{n}\left(\bar{X}^{h} \frac{\partial}{\partial \bar{x}^{h}}+\bar{X}^{\bar{h}} \frac{\partial}{\partial \bar{y}^{h}}\right)=\sum_{h=1}^{n}\left(X^{h} \frac{\partial}{\partial \bar{x}^{h}}+\partial X^{h} \frac{\partial}{\partial \bar{y}^{h}}\right)$, its components
isfy

$$
\sum_{k=1}^{n} R_{k j i}{ }^{h} \bar{X}^{k}=\sum_{k=1}^{n} R_{k j i}{ }^{h} X^{k}=0 \quad \text { and }
$$




$$
\sum_{k=1}^{n} R_{k j i}{ }^{h}\left(\bar{X}^{\bar{k}}-\partial \bar{X}^{k}\right)=0
$$

for all $h, i, j=1,2, \ldots, n$.

Hence, from Proposition 2, $X^{V}, X^{C} \in \Gamma(\bar{N})$.

The differential of the projection map $\pi$, written as $\pi$, will map the tangent bundle of $T M$ to $T M, \pi_{*}: T(T M) \rightarrow T M$. If $\bar{Y}$ is in $T(T M)$ at $(p, X)$, then $\pi_{*}$ will be a tangent at $p$ with $\left(\pi_{*} \bar{Y}\right) f=\bar{Y}(f \circ \pi)$ for all $f \in C^{\infty}(M)$. Since the nullity distribution $\bar{N}$ of $T M$ is a subspace of the tangent bundle of $T M$, we would like to see the effect of $\pi_{*}$ over $\bar{N}$.

Proposition 4. $N(p)=\pi_{*}(\bar{N}(p, X))$ for any $X \in T_{p} M$.

Proof. Let $\bar{Y}(p, X)=\sum_{A} \bar{Y}^{A}(p, X) \frac{\partial}{\partial \bar{x}^{A}}(p, X) \in \bar{N}(p, X)$. From Proposition 2 we have $\sum_{k=1}^{n} R_{k j i}{ }^{h} \bar{Y}^{k}(p, X)=0$ and $\sum_{k=1}^{n} R_{k j i}{ }^{h}\left(\bar{Y}^{\bar{k}}-\partial \bar{Y}^{k}\right)(p, X)=0$. Then $\pi_{*} \bar{Y}(p, X)=$ $\sum_{i=1}^{n} \bar{Y}^{i}(p, X) \frac{\partial}{\partial x^{i}}(p)$. By Proposition $1, \pi_{*}\left(\bar{Y}(p, X) \in N(p)\right.$. Therefore, $\pi_{*} \bar{N}(p, X) \subseteq$ $N(p)$. Together with Prposition 3, we have $N(p)=\pi_{*} \bar{N}(p, X)$.

\section{The Dimension of The Nullity Distribution on $\left(T M, g^{C}, \nabla^{C}\right)$}

From here on, we shall assume that the nullity distribution $N$ on $M$ is nonsingular.

Proposition 5. If the dimension of the nullity distribution $N$ on $(M, g)$ is $d$, then the dimension of the nullity distribution $\bar{N}$ on $\left(T M, g^{C}\right)$ is greater or equal to $2 d$.

Proof. Suppose that the dimension of the nullity distribution $N$ of $(M, g)$ is $d$. Let $\left(\xi_{1}, \xi_{2}, \ldots, \xi_{d}\right)$ be a local basis of $\Gamma(N)$. From Proposition 3 , we already know that $\xi_{i}^{V}, \xi_{i}^{C}$ are in $\Gamma(\bar{N})$ for all $i=1,2, \ldots, d$. Let $\xi_{i}=\sum_{j=1}^{n} \xi_{i}^{j} \frac{\partial}{\partial x^{j}}$. Since the dimension of $N$ is $d$, the rank of the matrix $\left(\xi_{i}^{j}\right)$ is also $d$. The set $\left\{\xi_{i}^{V}\right\}_{i=1}^{d}$ is linearly independent since $\xi_{i}^{V}=\sum_{j=1}^{n} \xi_{i}^{j} \frac{\partial}{\partial \bar{y}^{j}}$ and the rank of the matrix $\left(\xi_{i}^{j}\right)$ is $d$. Consider the linear equation $\sum_{i=1}^{d}\left(a^{i} \xi_{i}^{V}+b^{i} \xi_{i}^{C}\right)=0$. We weould like to show that this is true only if all $a^{i}, b^{i}=0$.

The equation becomes

$$
\begin{aligned}
\sum_{i=1}^{d}\left(a^{i} \xi_{i}^{V}+b^{i} \xi_{i}^{C}\right) & =\sum_{i=1}^{d} \sum_{j=1}^{n}\left(a^{i} \xi_{i}^{j} \frac{\partial}{\partial \bar{y}^{j}}+b^{i} \partial \xi \frac{\partial}{\partial \bar{y}^{j}}+b^{i} \xi_{i}^{j} \frac{\partial}{\partial \bar{x}^{j}}\right) \\
& =\sum_{i=1}^{d} \sum_{j=1}^{n}\left(\left(a^{i} \xi_{i}^{j}+b^{i} \partial \xi_{i}^{j}\right) \frac{\partial}{\partial \bar{y}^{j}}+b^{i} \xi_{i}^{j} \frac{\partial}{\partial \bar{x}^{j}}\right) \\
& =0
\end{aligned}
$$

Thus

$$
\begin{aligned}
\sum_{i=1}^{d} \sum_{j=1}^{n} b^{i} \xi_{i}^{j} \frac{\partial}{\partial \bar{x}^{j}} & =\sum_{i=1}^{d} b^{i}\left(\sum_{j=1}^{n} \xi_{i}^{j} \frac{\partial}{\partial \bar{x}^{j}}\right) \\
& =0
\end{aligned}
$$


Since $\left\{\xi_{i}\right\}_{i=1}^{d}$ is linearly independent, we have $b^{i}=0$ for all $i=1,2, \ldots, d$. Also

$$
\begin{aligned}
\sum_{i=1}^{d} \sum_{j=1}^{n}\left(a^{i} \xi_{i}^{j}+b^{i} \partial \xi_{i}^{j}\right) \frac{\partial}{\partial \bar{y}^{j}} & =\sum_{i=1}^{d} \sum_{j=1}^{n} a^{i} \xi_{i}^{j} \frac{\partial}{\partial \bar{y}^{j}} \\
& =\sum_{i=1}^{d} a^{i} \xi_{i}^{V} \\
& =0
\end{aligned}
$$

implies that $a^{i}=0$ for all $i=1,2, \ldots, d$ since $\left\{\xi_{i}^{V}\right\}_{i=1}^{d}$ is linearly independent.

Hence $\left\{\xi_{i}^{V}, \xi_{i}^{C}\right\}_{i=1}^{d}$ is linearly independent. This shows that the dimension of $\bar{N}$ is greater or equal to $2 \mathrm{~d}$.

We would like to show under the assumption that both $N$ and $\bar{N}$ are nonsingular, the dimension of $\bar{N}$ is actually $2 \mathrm{~d}$, where $d=\operatorname{dim} N$, i.e., at each point $\left(\bar{x}^{1}, \bar{x}^{2}, \ldots, \bar{x}^{n}, \bar{y}^{1}\right.$, $\left.\bar{y}^{2}, \ldots, \bar{y}^{n}\right), N_{\left(\bar{x}^{1}, \bar{x}^{2}, \ldots, \bar{x}^{n}, \bar{y}^{1}, \bar{y}^{2}, \ldots, \bar{y}^{n}\right)}$ is a $2 \mathrm{~d}$ dimension subspace of $T_{\left(\bar{x}^{1}, \bar{x}^{2}, \ldots, \bar{x}^{n}, \bar{y}^{1}, \bar{y}^{2}, \ldots, \bar{y}^{n}\right)}$

Prposition 6. If both the nullity distributions $N$ of $(M, g, \nabla)$ and $\bar{N}$ of $\left(T M, g^{C}, \nabla^{C}\right)$ are nonsingular, then the dimension of $\bar{N}$ is twice that of the dimension of $N$.

Proof. Let $d=\operatorname{dim} N$. For any $\bar{X} \in \Gamma(\bar{U}, \bar{N})$, let $\bar{X}^{A}, \bar{R}_{A B C}^{D}$ be the local components of $\bar{X}$ and $R^{C}$ respectively with respect to the induced coordinates.

The system (6) at a point $\left(\bar{x}^{1}, \bar{x}^{2}, \ldots, x^{n}, \bar{y}^{1}, \bar{y}^{2}, \ldots, \bar{y}^{n}\right)$ in $T M$ will be

$$
\left\{\begin{array}{r}
\left(\sum_{k=1}^{n} R_{k j i}{ }^{h} \bar{x}^{k}\right)\left(\bar{x}^{1}, \bar{x}^{2}, \ldots, x^{n}, \bar{y}^{1}, \bar{y}^{2}, \ldots, \bar{y}^{n}\right)=0 \\
\left(\sum_{k=1}^{n} R_{k j i}{ }^{h}\left(\bar{X}^{\bar{k}}-\partial \bar{X}^{k}\right)\right)\left(\bar{x}^{1}, \bar{x}^{2}, \ldots, x^{n}, \bar{y}^{1}, \bar{y}^{2}, \ldots, \bar{y}^{n}\right)=0
\end{array}\right\}
$$

$h, i, j=1,2, \ldots, n$. Its solutions will have the form $\left\{\bar{X}^{k}, \bar{X}^{\bar{k}}\right\}_{k=1}^{n}=\left\{\bar{X}^{k}, \bar{Y}^{k}+\partial \bar{X}^{k}\right\}_{k=1}^{n}$, where $\left\{\bar{X}^{k}\right\}_{k=1}^{n},\left\{\bar{Y}^{k}\right\}_{k=1}^{n}$ satisfy the first equation of (7).

Note that $R_{j k i}{ }^{h}\left(\bar{x}^{1}, \bar{x}^{2}, \ldots, x^{n}, \bar{y}^{1}, \bar{y}^{2}, \ldots, \bar{y}^{n}\right)$ are functions of $\left(\bar{x}^{1}, \bar{x}^{2}, \ldots, \bar{x}^{n}\right)$.

Since the degree of freedom of $\left\{\bar{X}^{k}\right\}_{k=1}^{n}$ is equal to $\operatorname{dim} N\left(\bar{x}^{1}, \bar{x}^{2}, \ldots, \bar{x}^{n}\right)=d$, we now have to show that the degree of freedom of $\left\{\bar{X}^{k}, \bar{Y}^{k}+\partial \bar{X}^{k}\right\}_{k=1}^{n}=\left\{\bar{Y}^{k}+\sum_{i=1}^{n} \bar{y}^{l} \frac{\partial \bar{X}^{k}}{\partial \bar{x}^{i}}\right\}_{k=1}^{n}$
is $d$.

From the assumption that $\bar{N}$ is nonsingular, we have

$$
\operatorname{dim} \bar{N}_{\left(\bar{x}^{1}, \bar{x}^{2}, \ldots, x^{n}, \bar{y}^{1}, \bar{y}^{2}, \ldots, \bar{y}^{n}\right)}=\operatorname{dim} \bar{N}_{\left(\bar{x}^{1}, \bar{x}^{2}, \ldots, \bar{x}^{n}, 0, \ldots, 0\right)} .
$$

Thus, we only need to show that $\left\{\left(\bar{Y}^{k}+\sum_{l=1}^{n} \bar{y}^{l} \frac{\partial \bar{X}^{k}}{\partial \bar{x}^{I}}\right)_{\left(\bar{x}^{1}, \bar{x}^{2}, \ldots, \bar{x}^{n}, 0, \ldots, 0\right)}\right\}_{k=1}^{n}$ is of degree
$d$. It is clear that

$$
\left\{\left(\bar{Y}^{k}+\sum_{l=1}^{n} \bar{y}^{l} \frac{\partial \bar{X}^{k}}{\partial \bar{x}^{l}}\right)_{\left(\bar{x}^{1}, \bar{x}^{2}, \ldots, \bar{x}^{n}, 0, \ldots, 0\right)}\right\}_{k=1}^{n}=\left\{\bar{Y}^{k}\left(\bar{x}^{1}, \bar{x}^{2}, \ldots, \bar{x}^{n}, 0, \ldots, 0\right)\right\}_{k=1}^{n} .
$$

The degree of freedom of $\left\{\bar{Y}^{k}\left(\bar{x}^{1}, \bar{x}^{2}, \ldots, \bar{x}^{n}, 0, \ldots, 0\right)\right\}_{k=1}^{n}$ is clearly $d$ since the second set of equations in (7) reduces to $\left(\sum_{k=1}^{n} R_{k j i}{ }^{h} \bar{Y}^{k}\right)\left(\bar{x}^{1}, \bar{x}^{2}, \ldots, \bar{x}^{n}, 0, \ldots, 0\right)=0$. 
Remark. If we assume only that $N$ is nonsingular, $\bar{N}$ may still be singular.

The results in this paper are contained in the author's M. Sc. thesis [2]. The author would like to express his gratitude to his supervisors Dr. S. L. Tan and Dr. S. H. Kon for their encouragement and guidance during this work.

\section{References}

[1] S. S. Chern and N. H. Kuiper, Some theorem on the isometric imbedding on compact riemannian manifolds in Euclidean spaces, Ann. of Math. 56(1952), 422-430.

[2] Wee-Keong Lim, Differential Geometry of Tangent Bundles of Riemannian Manifolds, M. Sc. theisis, Univeristy of Malaya, Kuala Lumpur, 1997.

[3] Sin-Leng Tan, On nullity distributions, Trans. Amer. Math. Soc. 223(1976), 323-335.

[4] Kentaro Yano and Shigeru Ishihara, Tangent and Cotangent Bundles, Marcel Dekker, New York, 1973.

Universiti Telekom, Jalan Ayer Keroh Lama, Bukit Beruang, 75450, Melaka, Malaysia. 